\title{
EXACT SOLUTION OF THE SIX-VERTEX MODEL WITH DOMAIN WALL BOUNDARY CONDITIONS. CRITICAL LINE BETWEEN FERROELECTRIC AND DISORDERED PHASES
}

\author{
PAVEL BLEHER AND KARL LIECHTY
}

\begin{abstract}
This is a continuation of the papers [4] of Bleher and Fokin and [6] of Bleher and Liechty, in which the large $n$ asymptotics is obtained for the partition function $Z_{n}$ of the six-vertex model with domain wall boundary conditions in the disordered and ferroelectric phases, respectively. In the present paper we obtain the large $n$ asymptotics of $Z_{n}$ on the critical line between these two phases.
\end{abstract}

\section{InTRODUCTION AND FORMULATION OF THE MAIN RESUlT}

1.1. Definition of the model. The six-vertex model, or the model of two-dimensional ice, is stated on a square $n \times n$ lattice with arrows on edges. The arrows obey the rule that at every vertex there are two arrows pointing in and two arrows pointing out. Such rule is sometimes called the ice-rule. There are only six possible configurations of arrows at each vertex, hence the name of the model, see Fig. 1.

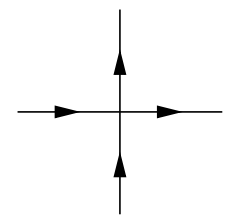

(1)

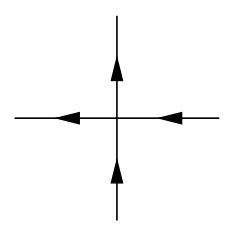

(4)

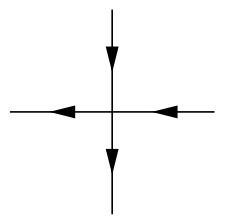

(2)

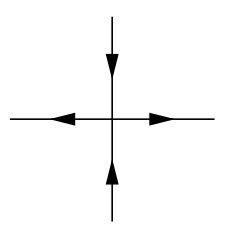

$(5)$

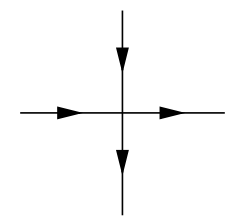

(3)

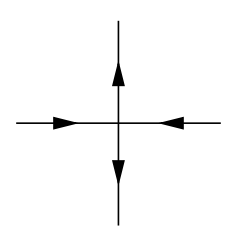

(6)

Figure 1. The six arrow configurations allowed at a vertex.

We will consider the domain wall boundary conditions (DWBC), in which the arrows on the upper and lower boundaries point in the square, and the ones on the left and right boundaries point out. One possible configuration with DWBC on the $4 \times 4$ lattice is shown on Fig. 2.

Date: October 29, 2018.

The first author is supported in part by the National Science Foundation (NSF) Grant DMS-0652005. 


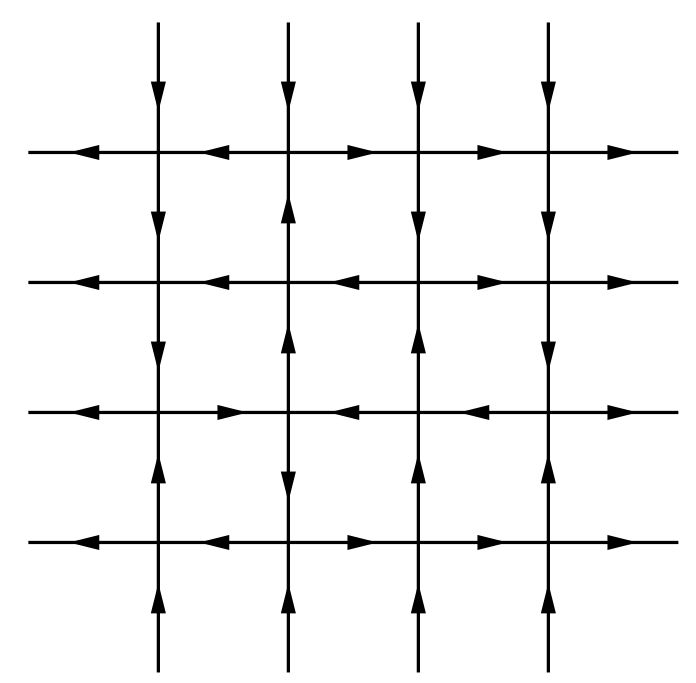

Figure 2. An example of $4 \times 4$ configuration with DWBC.

For each possible vertex state we assign a weight $w_{i}, i=1, \ldots, 6$, and define, as usual, the partition function, as a sum over all possible arrow configurations of the product of the vertex weights,

$$
Z_{n}=\sum_{\text {arrow configurations } \sigma} w(\sigma), \quad w(\sigma)=\prod_{x \in V_{n}} w_{\sigma(x)}=\prod_{i=1}^{6} w_{i}^{N_{i}(\sigma)},
$$

where $V_{n}$ is the $n \times n$ set of vertices, $\sigma(x) \in\{1, \ldots, 6\}$ is the vertex state of the configuration $\sigma$ at the vertex $x$, according to Figure 1 , and $N_{i}(\sigma)$ is the number of vertices of the vertex state $i$ in the configuration $\sigma$. The sum is taken over all possible configurations obeying the given boundary condition. The Gibbs measure is defined then as

$$
\mu_{n}(\sigma)=\frac{w(\sigma)}{Z_{n}}
$$

Our main goal is to obtain the large $n$ asymptotics of the partition function $Z_{n}$.

In general, the six-vertex model has six parameters: the weights $w_{i}$. However, by using some conservation laws we can reduce these to only two parameters. Namely, first we reduce to the case

$$
w_{1}=w_{2} \equiv a, \quad w_{3}=w_{4} \equiv b, \quad w_{5}=w_{6} \equiv c,
$$

and then, by using the identity,

$$
Z_{n}(a, a, b, b, c, c)=c^{n^{2}} Z_{n}\left(\frac{a}{c}, \frac{a}{c}, \frac{b}{c}, \frac{b}{c}, 1,1\right)
$$

to the two parameters, $\frac{a}{c}$ and $\frac{b}{c}$. For details on how we make this reduction, see, e.g., the works [1] of Allison and Reshetikhin, [12] of Ferrari and Spohn, or the work [6].

1.2. The phase diagram. Introduce the parameter

$$
\Delta=\frac{a^{2}+b^{2}-c^{2}}{2 a b} \text {. }
$$


The phase diagram of the six-vertex model consists of the following three regions: the ferroelectric phase region, $\Delta>1$; the anti-ferroelectric phase region, $\Delta<-1$; and, the disordered phase region, $-1<\Delta<1$, see, e.g., [24]. In these three regions we parameterize the weights in the standard way: in the ferroelectric phase region,

$$
a=\sinh (t-\gamma), \quad b=\sinh (t+\gamma), \quad c=\sinh (2|\gamma|), \quad 0<|\gamma|<t,
$$

in the anti-ferroelectric phase region,

$$
a=\sinh (\gamma-t), \quad b=\sinh (\gamma+t), \quad c=\sinh (2 \gamma), \quad|t|<\gamma,
$$

and in the disordered phase region,

$$
a=\sin (\gamma-t), \quad b=\sin (\gamma+t), \quad c=\sin (2 \gamma), \quad|t|<\gamma .
$$

The phase diagram of the six-vertex model is shown in Fig. 3.

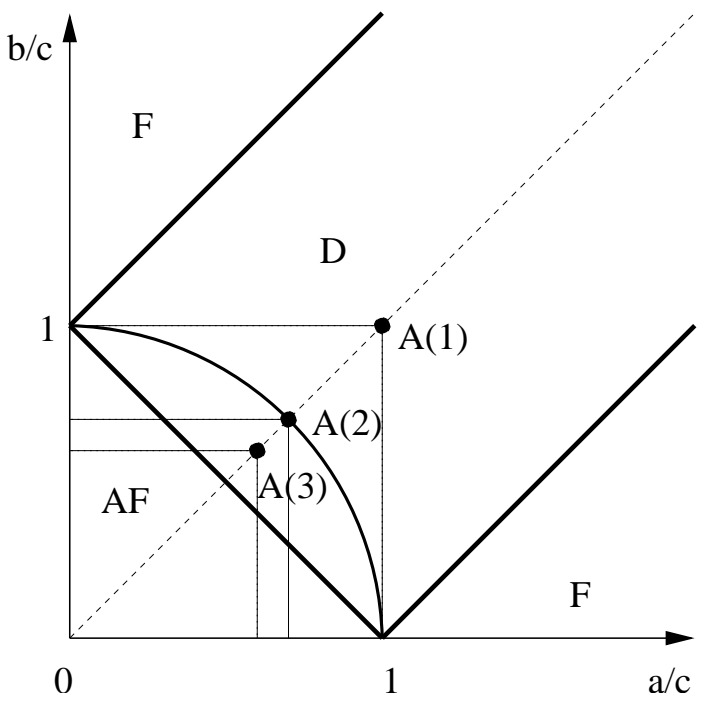

Figure 3. The phase diagram of the model, where $\mathbf{F}, \mathbf{A F}$ and $\mathbf{D}$ mark ferroelectric, antiferroelectric, and disordered phase regions, respectively. The circular arc corresponds to the so-called "free fermion" line, when $\Delta=0$, and the three dots correspond to 1-, 2-, and 3-enumeration of alternating sign matrices.

The phase diagram and the Bethe-Ansatz solution of the six-vertex model for periodic and anti-periodic boundary conditions are thoroughly discussed in the works of Lieb [20]-[23], Lieb, Wu [24], Sutherland [27], Baxter [2], Batchelor, Baxter, O’Rourke, Yung [3]. See also the work of $\mathrm{Wu}$, Lin [29], in which the Pfaffian solution for the six-vertex model with periodic boundary conditions is obtained on the free fermion line, $\Delta=0$.

As concerns the six-vertex model with DWBC, it is noticed by Kuperberg [19], that on the diagonal,

$$
\frac{a}{c}=\frac{b}{c}=x
$$

the six-vertex model with DWBC is equivalent to the $s$-enumeration of alternating sign matrices (ASM), in which the weight of each such matrix is equal to $s^{N_{-}}$, where $N_{-}$is the 
number of $(-1)$ 's in the matrix and $s=\frac{1}{x^{2}}$. The exact solution for a finite $n$ is known for 1-, 2-, and 3-enumerations of ASMs, see the works by Kuperberg [19] and Colomo and Pronko [9] for a solution based on the Izergin-Korepin formula. A fascinating story of the discovery of the ASM formula is presented in the book [7] of Bressoud. On the free fermion line, $\gamma=\frac{\pi}{4}$, the partition function of the six-vertex model with DWBC has a very simple form: $Z_{n}=1$. For a nice short proof of this formula see the work [9] of Colomo and Pronko.

Here we will discuss the ferroelectric and disordered phase regions, and we will use parameterizations (1.6) and (1.8). The ferroelectric phase region consists of two connected components. For the sake of concreteness we will assume that

$$
\gamma>0
$$

which corresponds to the component where

$$
b>a+c .
$$

The parameter $\Delta$ in the ferroelectric phase region reduces to

$$
\Delta=\cosh (2 \gamma) \text {. }
$$

1.3. Exact solution of $Z_{n}$ for finite $n$ in ferroelectric phase. The six-vertex model with DWBC was first introduced by Korepin in [16]. In this paper, he derived an important recursion relation for $Z_{n}$, which was subsequently used by Izergin [13] in deriving a determinantal formula for $Z_{n}$ in this model. A detailed proof of this formula and its generalizations are given in the paper of Izergin, Coker, and Korepin [14], see also the papers of Korepin, Zinn-Justin [18] and Kuperberg [19] and the book of Bressoud [7]. When the weights are parameterized according to (1.6), the formula of Izergin-Korepin is

$$
Z_{n}=\frac{[\sinh (t-\gamma) \sinh (t+\gamma)]^{n^{2}}}{\left(\prod_{j=0}^{n-1} j !\right)^{2}} \tau_{n}
$$

where $\tau_{n}$ is the Hankel determinant,

$$
\tau_{n}=\operatorname{det}\left(\frac{d^{j+k-2} \phi}{d t^{j+k-2}}\right)_{1 \leq j, k \leq n}
$$

and

$$
\phi(t)=\frac{\sinh (2 \gamma)}{\sinh (t+\gamma) \sinh (t-\gamma)}
$$

The aspect of the Izergin-Korepin determinantal formula that we exploit in this paper is that $\tau_{N}$ can be expressed in terms of related orthogonal polynomials, see the paper [30] of Zinn-Justin. In the ferroelectric phase the expression in terms of orthogonal polynomials can be obtained in the following manner. For the evaluation of the Hankel determinant, let us write $\phi(t)$ in the form of the Laplace transform of a discrete measure,

$$
\phi(t)=\frac{\sinh (2 \gamma)}{\sinh (t+\gamma) \sinh (t-\gamma)}=4 \sum_{l=1}^{\infty} e^{-2 t l} \sinh (2 \gamma l)
$$

Then

$$
\tau_{n}=\frac{2^{n^{2}}}{n !} \sum_{l_{1}, \ldots, l_{n}=1}^{\infty} \Delta\left(l_{i}\right)^{2} \prod_{i=1}^{n}\left[2 e^{-2 t l_{i}} \sinh \left(2 \gamma l_{i}\right)\right]
$$


where

$$
\Delta\left(l_{i}\right)=\prod_{1 \leq i<j \leq n}\left(l_{j}-l_{i}\right)
$$

is the Vandermonde determinant.

Introduce now discrete monic polynomials $P_{j}(x)=x^{j}+\ldots$ orthogonal on the set $\mathbb{N}=$ $\{l=1,2, \ldots\}$ with respect to the weight,

$$
w(l)=2 e^{-2 t l} \sinh (2 \gamma l)=e^{-2 t l+2 \gamma l}-e^{-2 t l-2 \gamma l},
$$

so that

$$
\sum_{l=1}^{\infty} P_{j}(l) P_{k}(l) w(l)=h_{k} \delta_{j k}
$$

Then it follows from (1.17) that

$$
\tau_{n}=2^{n^{2}} \prod_{k=0}^{n-1} h_{k}
$$

see Appendix in the end of the paper [6].

1.4. Exact solution of $Z_{n}$ for finite $n$ on the critical line between the ferroelectric and disordered phases. We consider the partition function $Z_{n}$ on the critical line

$$
\frac{b}{c}-\frac{a}{c}=1
$$

We fix a point,

$$
\frac{a}{c}=\frac{\alpha-1}{2}, \quad \frac{b}{c}=\frac{\alpha+1}{2} ; \quad \alpha>1,
$$

on this line, and we are interested in the large $n$ asymptotics of the partition function

$$
Z_{n}=Z_{n}\left(\frac{\alpha-1}{2}, \frac{\alpha-1}{2}, \frac{\alpha+1}{2}, \frac{\alpha+1}{2}, 1,1\right) .
$$

Let us first derive a formula for $Z_{n}$ on the critical line. To that end, consider the limit in the Izergin-Korepin formula in the ferroelectric phase, (1.13), as

$$
t, \gamma \rightarrow+0, \quad \frac{t}{\gamma} \rightarrow \alpha
$$

Observe that in this limit,

$$
\frac{a}{c}=\frac{\sinh (t-\gamma)}{\sinh (2 \gamma)} \rightarrow \frac{\alpha-1}{2}, \quad \frac{b}{c}=\frac{\sinh (t+\gamma)}{\sinh (2 \gamma)} \rightarrow \frac{\alpha+1}{2} .
$$

By (1.4),

$$
Z_{n}\left(\frac{a}{c}, \frac{a}{c}, \frac{b}{c}, \frac{b}{c}, 1,1\right)=\frac{Z_{n}(a, a, b, b, c, c)}{c^{n^{2}}},
$$

hence by (1.13), and (1.21),

$$
Z_{n}\left(\frac{a}{c}, \frac{a}{c}, \frac{b}{c}, \frac{b}{c}, 1,1\right)=\left[\frac{2 \sinh (t-\gamma) \sinh (t+\gamma)}{\sinh (2 \gamma)}\right]^{n^{2}} \prod_{k=0}^{n-1} \frac{h_{k}}{(k !)^{2}}
$$


To deal with limit (1.25) we need to rescale the orthogonal polynomials $P_{k}(l)$. Introduce the rescaled variable,

$$
x=2 t l-2 \gamma l
$$

and the rescaled limiting weight,

$$
w_{\alpha}(x)=\lim _{t, \gamma \rightarrow+0, \frac{t}{\gamma} \rightarrow \alpha}\left(e^{-2 t l+2 \gamma l}-e^{-2 t l-2 \gamma l}\right)=e^{-x}-e^{-r x}, \quad r=\frac{\alpha+1}{\alpha-1}>1 .
$$

Consider monic orthogonal polynomials $P_{j}(x ; \alpha)$ satisfying the orthogonality condition,

$$
\int_{0}^{\infty} P_{j}(x ; \alpha) P_{k}(x ; \alpha) w_{\alpha}(x) d x=h_{k, \alpha} \delta_{j k} .
$$

To find a relation between $P_{k}(l)$ and $P_{k}(x ; \alpha)$, introduce the monic polynomials

$$
\tilde{P}_{k}(x)=\Delta^{k} P_{k}(x / \Delta) \text {, }
$$

where

$$
\Delta=2 t-2 \gamma
$$

and rewrite orthogonality condition (1.20) in the form

$$
\sum_{l=1}^{\infty} \tilde{P}_{j}(l \Delta) \tilde{P}_{k}(l \Delta) w_{\alpha}(l \Delta) \Delta=\Delta^{2 k+1} h_{k} \delta_{j k}
$$

which is a Riemann sum for the integral in orthogonality condition (1.31). Therefore,

$$
\lim _{t, \gamma \rightarrow+0, \frac{t}{\gamma} \rightarrow \alpha} \tilde{P}_{k}(x)=P_{k}(x ; \alpha),
$$

and

$$
\lim _{t, \gamma \rightarrow+0, \frac{t}{\gamma} \rightarrow \alpha} \Delta^{2 k+1} h_{k}=h_{k, \alpha} .
$$

Let us rewrite formula (1.28) as

$$
Z_{n}\left(\frac{a}{c}, \frac{a}{c}, \frac{b}{c}, \frac{b}{c}, 1,1\right)=\left[\frac{2 \sinh (t-\gamma) \sinh (t+\gamma)}{\sinh (2 \gamma) \Delta}\right]^{n^{2}} \prod_{k=0}^{n-1} \frac{\Delta^{2 k+1} h_{k}}{(k !)^{2}}
$$

and take limit (1.25). In the limit we obtain that

$$
Z_{n}=Z_{n}\left(\frac{\alpha-1}{2}, \frac{\alpha-1}{2}, \frac{\alpha+1}{2}, \frac{\alpha+1}{2}, 1,1\right)=\left(\frac{\alpha+1}{2}\right)^{n^{2}} \prod_{k=0}^{n-1} \frac{h_{k, \alpha}}{(k !)^{2}}, .
$$

Our main technical result in this paper will be the proof of the following asymptotics of $h_{k, \alpha}$. Let, as usual,

$$
\zeta(s)=1+\frac{1}{2^{s}}+\frac{1}{3^{s}}+\ldots, \quad \operatorname{Re} s>1 .
$$

Theorem 1.1. As $k \rightarrow \infty$,

$$
\ln \left[\frac{h_{k, \alpha}}{(k !)^{2}}\right]=-\frac{\zeta\left(\frac{3}{2}\right)}{2 \sqrt{\pi(r-1)} k^{1 / 2}}+\frac{1}{4 k}+O\left(k^{-3 / 2}\right), \quad r=\frac{\alpha+1}{\alpha-1} .
$$

A proof of this theorem will be given below. 
1.5. Main result. This paper is a continuation of the works [4] and [6], in which the large $n$ asymptotics of $Z_{n}$ is obtained in the disordered and ferroelectric phase, respectively. In [4] it is proven that in the disordered phase, for some $\varepsilon>0$, as $n \rightarrow \infty$,

$$
Z_{n}\left(\frac{a}{c}, \frac{a}{c}, \frac{b}{c}, \frac{b}{c}, 1,1\right)=C n^{\kappa} F^{n^{2}}\left[1+O\left(n^{-\varepsilon}\right)\right],
$$

where in parameterization (1.8),

$$
\kappa=\frac{1}{12}-\frac{2 \gamma^{2}}{3 \pi(\pi-\gamma)}
$$

and

$$
F=\frac{\pi \sin (\gamma-t) \sin (\gamma+t)}{2 \gamma \sin (2 \gamma) \cos \frac{\pi t}{2 \gamma}}
$$

The value of the constant $C$ in (1.41) is not yet known. In [6] it is proven that in the ferroelectric phase, for any $\varepsilon>0$, as $n \rightarrow \infty$,

$$
Z_{n}\left(\frac{a}{c}, \frac{a}{c}, \frac{b}{c}, \frac{b}{c}, 1,1\right)=C G^{n} F^{n^{2}}\left[1+O\left(e^{-n^{1-\varepsilon}}\right)\right],
$$

where in parameterization (1.6),

$$
C=1-e^{-4|\gamma|}, \quad G=e^{|\gamma|-t},
$$

and

$$
F=\frac{\sinh (t+\gamma)}{\sinh (2 \gamma)}
$$

The main result of this paper is the following asymptotics of $Z_{n}$ on the critical line between these two phases.

Theorem 1.2. As $n \rightarrow \infty$,

$$
Z_{n}\left(\frac{\alpha-1}{2}, \frac{\alpha-1}{2}, \frac{\alpha+1}{2}, \frac{\alpha+1}{2}, 1,1\right)=C n^{\kappa} G^{\sqrt{n}} F^{n^{2}}\left[1+O\left(n^{-1 / 2}\right)\right],
$$

where $C>0$,

$$
\kappa=\frac{1}{4}, \quad G=\exp \left[-\zeta\left(\frac{3}{2}\right) \sqrt{\frac{\alpha-1}{2 \pi}}\right]
$$

and

$$
F=\frac{\alpha+1}{2}
$$


The proof of Theorem 1.2 follows easily from Theorem 1.1. Namely, from formula (1.38) and asymptotics (1.40) we obtain that

$$
\begin{aligned}
\ln & {\left[\frac{Z_{n}\left(\frac{\alpha-1}{2}, \frac{\alpha-1}{2}, \frac{\alpha+1}{2}, \frac{\alpha+1}{2}, 1,1\right)}{\left(\frac{\alpha+1}{2}\right)^{n^{2}}}\right]=\sum_{k=0}^{n-1} \ln \left[\frac{h_{k, \alpha}}{(k !)^{2}}\right] } \\
& =\sum_{k=0}^{n-1}\left[-\frac{\zeta\left(\frac{3}{2}\right)}{2 \sqrt{\pi(r-1)} k^{1 / 2}}+\frac{1}{4 k}+O\left(k^{-3 / 2}\right)\right] \\
& =-\zeta\left(\frac{3}{2}\right) \sqrt{\frac{(\alpha-1)}{2 \pi}} n^{1 / 2}+\frac{\ln n}{4}+C_{0}+O\left(n^{-1 / 2}\right),
\end{aligned}
$$

which implies Theorem 1.2 .

1.6. Ground state configuration on the critical line. The ground state configuration $\sigma^{\text {gs }}$ has the maximal weight. On the upper critical line between the ferroelectric and disordered phase regions we have that $b=a+c$. We also assume that $a>0, c>0$, hence $\sigma^{\text {gs }}$ should contain as many $b$ 's as possible. The domain wall boundary conditions imply that in each row there is at least one $c$. Therefore, any weight cannot be bigger than $b^{n^{2}-n} c^{n}$. The weight $b^{n^{2}-n} c^{n}$ does occur for the following unique configuration:

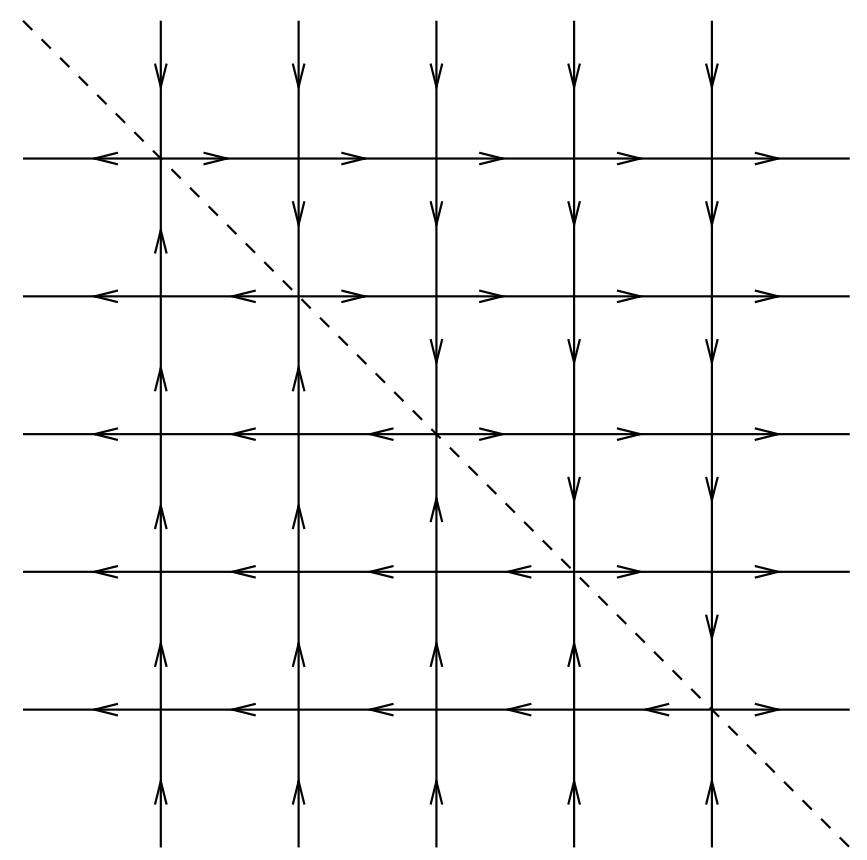

Figure 4. A ground state configuration.

$$
\sigma^{\mathrm{gs}}(x)= \begin{cases}\sigma_{5} & \text { if } x \text { is on the diagonal, } \\ \sigma_{3} & \text { if } x \text { is above the diagonal, } \\ \sigma_{4} & \text { if } x \text { is below the diagonal, }\end{cases}
$$


see Fig. 4, which is the unique ground state configuration with domain wall boundary conditions on the critical line. In the ferroelectric phase region, where $b>a+c$, the ground state configuration is obviously the same. If we set

$$
a=\frac{\alpha-1}{2}, \quad b=\frac{\alpha+1}{2}, \quad c=1,
$$

then the weight of the ground state configuration is equal to

$$
w\left(\sigma^{\mathrm{gs}}\right)=F^{n^{2}-n}, \quad F=\frac{\alpha+1}{2} .
$$

By (1.47) the ratio $Z_{n} / w\left(\sigma^{\mathrm{gs}}\right)$ is evaluated as

$$
\frac{Z_{n}}{w\left(\sigma^{\mathrm{gs}}\right)}=C n^{\kappa} G^{\sqrt{n}} F^{n}\left[1+O\left(n^{-1 / 2}\right)\right] .
$$

Observe that

$$
\lim _{n \rightarrow \infty} \frac{\ln Z_{n}}{n^{2}}=\lim _{n \rightarrow \infty} \frac{\ln w\left(\sigma^{\mathrm{gs}}\right)}{n^{2}}=\ln F
$$

so that the free energy is determined by the free energy of the ground state configuration. This can be explained by the fact that low energy excited states are local perturbations of the ground state around the diagonal. Namely, it is impossible to create a new configuration by perturbing the ground state locally away of the diagonal: the conservation law $N_{3}(\sigma)=N_{4}(\sigma)$ forbids such a configuration. Therefore, typical configurations of the six-vertex model in the ferroelectric phase region and on the critical line between the ferroelectric and disordered phase regions are frozen outside of a relatively small neighborhood of the diagonal.

This behavior of typical configurations in the ferroelectric phase region and on the critical line between the ferroelectric and disordered phase regions is in a big contrast with the situation in the disordered and anti-ferroelectric phase regions. Extensive rigorous, theoretical and numerical studies, see, e.g., the works of Cohn, Elkies, Propp [8], Eloranta [11], Syljuasen, Zvonarev [26], Allison, Reshetikhin [1], Kenyon, Okounkov [15], Kenyon, Okounkov, Sheffield [17], Sheffield [25], Ferrari, Spohn [12], Colomo, Pronko [10], Zinn-Justin [31], and references therein, show that in the disordered and anti-ferroelectric phase regions the "arctic circle" phenomenon persists, so that there are macroscopically big frozen and random domains in typical configurations, separated in the limit $n \rightarrow \infty$ by an "arctic curve".

\section{LARGE $k$ ASYMPTOTICS OF $h_{k, \alpha}$}

We will use asymptotic formulae for orthogonal polynomials on $[0, \infty)$, obtained in the paper [28] of Vanlessen. To formulate and to apply the Vanlessen's asymptotic formula we will need to introduce some notations and to evaluate some parameters. Let us write

$$
w_{\alpha}(z)=e^{-z}-e^{-r z}=z e^{-Q(z)},
$$

so that

$$
Q(z)=z+\log \frac{z}{1-e^{-(r-1) z}}
$$

where for $\log$ we take the principal branch with a cut at $(-\infty, 0]$. Observe that the function $Q(z)$ is analytic in a strip $|\operatorname{Im} z| \leq c_{0}, c_{0}>0$. Define the Mashkar-Rakhmanov-Saff numbers 
$\beta_{k}=\beta_{k}(\alpha)$ as a solution to the equation

$$
\frac{1}{2 \pi} \int_{0}^{\beta_{k}} Q^{\prime}(x) \sqrt{\frac{x}{\beta_{k}-x}} d x=k
$$

As shown in [28], for large $k$ there is a unique solution to this equation.

2.1. Evaluation of $\beta_{k}$. By the change of variable, $x=\beta_{k} u$, equation (2.3) reduces to

$$
\frac{\beta_{k}}{2 \pi} \int_{0}^{1} Q^{\prime}\left(\beta_{k} u\right) \sqrt{\frac{u}{1-u}} d u=k .
$$

Set

$$
b_{k}=\frac{\beta_{k}}{4 k}
$$

Then equation (2.4) reduces to

$$
\frac{2 b_{k}}{\pi} \int_{0}^{1} Q^{\prime}\left(4 b_{k} k u\right) \sqrt{\frac{u}{1-u}} d u=1
$$

From (2.2),

$$
Q^{\prime}(z)=1+\frac{1}{z}-\frac{r-1}{e^{(r-1) z}-1} .
$$

Observe that the function $Q^{\prime}(z)$ has poles at the points

$$
z=\frac{2 m \pi i}{r-1}, \quad m= \pm 1, \pm 2, \ldots
$$

After evaluating integrals of the first two terms of $Q^{\prime}\left(4 b_{k} k u\right)$, equation (2.6) reads

$$
b_{k}+\frac{1}{2 k}-\frac{2}{\pi} \int_{0}^{1} \frac{(r-1) b_{k}}{e^{4(r-1) b_{k} k u}-1} \sqrt{\frac{u}{1-u}} d u=1 .
$$

By the change of variable $x=k u$, it reduces to

$$
b_{k}+\frac{1}{2 k}-\frac{2}{\pi k^{3 / 2}} \int_{0}^{k} \frac{(r-1) b_{k}}{e^{4(r-1) b_{k} x}-1} \sqrt{\frac{x}{1-(x / k)}} d x=1 .
$$

Set

$$
\varepsilon=\frac{1}{k^{1 / 2}}
$$

and consider the function,

$$
f(b, \varepsilon)=b+\frac{\varepsilon^{2}}{2}-\frac{2 \varepsilon^{3}}{\pi} \int_{0}^{1 / \varepsilon^{2}} \frac{(r-1) b}{e^{4(r-1) b x}-1} \sqrt{\frac{x}{1-\varepsilon^{2} x}} d x-1, \quad \frac{1}{2} \leq b \leq 2 .
$$

Observe that as $\varepsilon \rightarrow 0$,

$$
\begin{aligned}
\int_{0}^{1 / \varepsilon^{2}} \frac{(r-1) b}{e^{4(r-1) b x}-1} \sqrt{\frac{x}{1-\varepsilon^{2} x}} d x & =\int_{0}^{\infty} \frac{(r-1) b \sqrt{x} d x}{e^{4(r-1) b x}-1}+O\left(\varepsilon^{2}\right) \\
& =\frac{\sqrt{\pi} \zeta\left(\frac{3}{2}\right)}{16 \sqrt{b(r-1)}}+O\left(\varepsilon^{2}\right),
\end{aligned}
$$


hence

$$
f(b, \varepsilon)=(b-1)+\frac{\varepsilon^{2}}{2}-\frac{\varepsilon^{3} \zeta\left(\frac{3}{2}\right)}{8 \sqrt{\pi b(r-1)}}+O\left(\varepsilon^{5}\right) .
$$

It is easy to see that equation (2.13) can be differentiated in $b$ infinitely many times, and hence the function $f(b, \varepsilon)$ is $C^{\infty}$ in a neighborhood of the point $b=1, \varepsilon=0$. In addition,

$$
f(1,0)=0, \quad \frac{\partial f(1,0)}{\partial b}=1 .
$$

By the implicit function theorem, there is a $C^{\infty}$-solution $b(\varepsilon)$ of the equation $f(b, \varepsilon)=0$. From (2.14) we obtain that

$$
b(\varepsilon)=1-\frac{\varepsilon^{2}}{2}+\frac{\varepsilon^{3} \zeta\left(\frac{3}{2}\right)}{8 \sqrt{\pi(r-1)}}+O\left(\varepsilon^{5}\right), \quad \varepsilon \rightarrow 0 .
$$

Since $b_{k}=b\left(k^{-1 / 2}\right)$, this gives

$$
b_{k}=1-\frac{1}{2 k}+\frac{\zeta\left(\frac{3}{2}\right)}{8 \sqrt{\pi(r-1)} k^{3 / 2}}+O\left(k^{-5 / 2}\right), \quad k \rightarrow \infty .
$$

By (2.5),

$$
\beta_{k}=4 k b_{k}
$$

2.2. Evaluation of the equilibrium measure. Set

$$
V_{k}(x)=\frac{1}{k} Q\left(\beta_{k} x\right)
$$

and consider the following minimization problem:

$$
E=\inf _{\mu} I(\mu)
$$

where

$$
I(\mu)=-\iint \log |x-y| d \mu(x) d \mu(y)+\int V_{k}(x) d \mu(x) .
$$

and inf is taken over all probability measures on $[0, \infty)$. There exists a unique minimizer, $\mu=\mu_{k}$, and it has the following properties:

(1) The support of $\mu_{k}$ is the interval $[0,1]$.

(2) The measure $\mu_{k}$ is absolutely continuous with respect to the Lebesgue measure.

(3) The density function of $\mu_{k}$ has the form,

$$
\frac{d \mu_{k}(x)}{d x} \equiv \psi_{k}(x)=\frac{1}{2 \pi} \sqrt{\frac{1-x}{x}} q_{k}(x),
$$

where $q_{k}(x)$ is analytic and positive on $[0,1]$.

The equilibrium measure $\mu_{k}$ is characterized by the Euler-Lagrange variational conditions: there exists $l_{k} \in \mathbb{R}$ such that

$$
\begin{aligned}
& 2 \int_{0}^{1} \log |x-y| d \mu_{k}(y)-V_{k}(x)-l_{k}=0 \quad \text { for } x \in[0,1], \\
& 2 \int_{0}^{1} \log |x-y| d \mu_{k}(y)-V_{k}(x)-l_{k} \leq 0 \quad \text { for } x \notin[0,1] .
\end{aligned}
$$


The function $q_{k}(z)$ in $(2.22)$ is given by the formula,

$$
q_{k}(z)=\frac{1}{2 \pi i} \oint_{\Gamma} \sqrt{\frac{y}{y-1}} \frac{V_{k}^{\prime}(y) d y}{y-z}, \quad z \in \operatorname{Int} \Gamma,
$$

where $\sqrt{\frac{y}{y-1}}$ is taken on the principal branch, with cut on $[0,1]$, and $\Gamma$ is a positively oriented contour containing $[0,1] \cup\{z\}$ in its interior, with the additional condition, that the function $V_{k}^{\prime}(y)$ is analytic inside $\Gamma$. By (2.19) and (2.2),

$$
V_{k}(z)=4 b_{k} z+\frac{1}{k} \ln \frac{4 b_{k} k z}{1-e^{-4(r-1) b_{k} k z}}
$$

hence

$$
V_{k}^{\prime}(z)=4 b_{k}+\frac{1}{k z}-\frac{\gamma_{k}}{e^{\gamma_{k} k z}-1}
$$

where

$$
\gamma_{k}=4(r-1) b_{k}
$$

hence

$$
q_{k}(z)=\frac{1}{2 \pi i} \oint_{\Gamma} \sqrt{\frac{y}{y-1}}\left[4 b_{k}+\frac{1}{k y}-\frac{\gamma_{k}}{e^{\gamma_{k} k y}-1}\right] \frac{d y}{y-z} .
$$

By taking the residue at infinity, we obtain that

$$
q_{k}(z)=4 b_{k}+s_{k}(z), \quad s_{k}(z)=-\frac{\gamma_{k}}{2 \pi i} \oint_{\Gamma} \sqrt{\frac{y}{y-1}} \frac{d y}{\left(e^{\gamma_{k} k y}-1\right)(y-z)} .
$$

Observe that the function $V_{k}^{\prime}(z)$ has poles at the points

$$
z_{n}=\frac{2 \pi n i}{\gamma_{k} k}, \quad n= \pm 1, \pm 2, \ldots
$$

hence the contour $\Gamma$ has to pass close to 0 . We choose $\Gamma$ such that

$$
\frac{c_{1}}{k} \geq \operatorname{dist}(0, \Gamma) \geq \frac{c_{2}}{k}, \quad c_{1} \geq \operatorname{dist}(1, \Gamma) \geq c_{2}>0,
$$

see Fig. 5. More precisely, let for a given $z \in \mathbb{C}, m(z) \in[0,1]$ be the closest point from $z$ on $[0,1]$, so that

$$
\inf \{|z-u|, u \in[0,1]\}=|z-m(z)|
$$

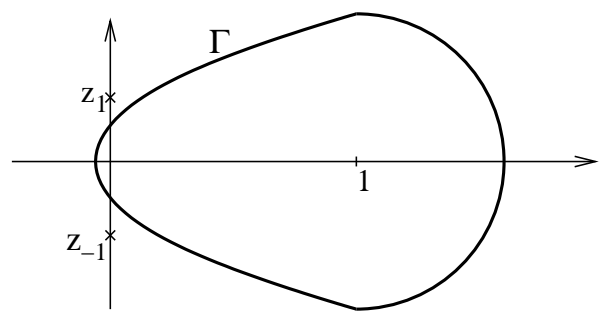

Figure 5. The contour $\Gamma$. 
Then we define, for a given $\delta>0$,

$$
\Gamma=\Gamma(\delta, k)=\left\{z \in \mathbb{C}:|z-m(z)|=\delta\left[\frac{1}{k}+m(z)\right],\right.
$$

and we choose $\delta$ to be sufficiently small so that the points $z_{n}$ in (2.30) lie outside of $\Gamma$, see Fig. 5. Observe that $\Gamma(0, k)=[0,1]$.

With the help of the change of variables, $u=k y$, we obtain that

$$
s_{k}(z)=-\frac{\gamma_{k}}{2 \pi i k^{1 / 2}} \oint_{k \Gamma} \sqrt{\frac{u}{(u / k)-1}} \frac{d u}{\left(e^{\gamma_{k} u}-1\right)(u-k z)},
$$

which implies that

$$
\sup _{0 \leq z \leq 1}\left|s_{k}(z)\right|=O\left(k^{-1 / 2}\right)
$$

or even that

$$
\sup _{0 \leq d \leq \frac{\delta}{2}} \sup _{z \in \Gamma(d, k)}\left|s_{k}(z)\right|=O\left(k^{-1 / 2}\right) .
$$

For $z>1$, the function $s_{k}(z)$ can be reduced to

$$
s_{k}(z)=-\gamma_{k} \sqrt{\frac{z}{z-1}} \frac{1}{\left(e^{\gamma_{k} k z}-1\right)}-\frac{\gamma_{k}}{\pi} \int_{0}^{1} \sqrt{\frac{y}{1-y}} \frac{d y}{\left(e^{\gamma_{k} k y}-1\right)(z-y)} .
$$

It implies that

$$
s_{k}(z)=\frac{a_{k}}{z}+r_{k}(z)
$$

where

$$
a_{k}=-\frac{\gamma_{k}}{\pi} \int_{0}^{1} \sqrt{\frac{y}{1-y}} \frac{d y}{\left(e^{\gamma_{k} k y}-1\right)}
$$

and $r_{k}(z)$ satisfies the estimate,

$$
\left|r_{k}(z)\right| \leq \frac{C}{z \sqrt{z-1} k^{5 / 2}}, \quad z>1
$$

with some $C>0$. Indeed,

$$
r_{k}(z)=-\gamma_{k} \sqrt{\frac{z}{z-1}} \frac{1}{e^{\gamma_{k} k z}-1}-\frac{\gamma_{k}}{\pi} \int_{0}^{1} \sqrt{\frac{y}{1-y}} \frac{y d y}{\left(e^{\gamma_{k} k y}-1\right)(z-y) z} .
$$

The first term on the right is exponentially small in $z$ and $k$, and it obviously satisfies estimate (2.40). In the second term on the right, let us split the integral in two integrals, from 0 to $\frac{1}{2}$ and from $\frac{1}{2}$ to 1 . The first part is estimated as follows:

$$
\begin{aligned}
0 & \leq \int_{0}^{\frac{1}{2}} \sqrt{\frac{y}{1-y}} \frac{y d y}{\left(e^{\gamma_{k} k y}-1\right)(z-y) z} \leq \frac{4}{z^{2}} \int_{0}^{\frac{1}{2}} \frac{y^{3 / 2} d y}{\left(e^{\gamma_{k} k y}-1\right)} \\
& \leq \frac{4}{z^{2} k^{5 / 2}} \int_{0}^{\infty} \frac{u^{3 / 2} d u}{\left(e^{\gamma_{k} u}-1\right)} \leq \frac{C_{0}}{z^{2} k^{5 / 2}}, \quad u=k y
\end{aligned}
$$


hence it satisfies estimate (2.40). For the second part we have that

$$
\begin{aligned}
0 & \leq \int_{\frac{1}{2}}^{1} \sqrt{\frac{y}{1-y}} \frac{y d y}{\left(e^{\gamma_{k} k y}-1\right)(z-y) z} \leq \frac{1}{z\left(e^{\gamma_{k} k / 2}-1\right)} \int_{\frac{1}{2}}^{1} \frac{d y}{(z-y) \sqrt{1-y}} \\
& =\frac{1}{z\left(e^{\gamma_{k} k / 2}-1\right)} \int_{0}^{\frac{1}{2}} \frac{d u}{(z-1+u) \sqrt{u}} \leq \frac{C_{1}}{z \sqrt{z-1}\left(e^{\gamma_{k} k / 2}-1\right)}, \quad u=1-y,
\end{aligned}
$$

which satisfies estimate (2.40). Thus, (2.40) is proved.

From (2.38) we obtain that

$$
q_{k}(z)=4 b_{k}+\frac{a_{k}}{z}+r_{k}(z)
$$

where $a_{k}$ is given by formula (2.39) and $r_{k}(z)$ satisfies estimate (2.40).

2.3. Evaluation of the Lagrange multiplier. Introduce the function

$$
g_{k}(z)=\int_{0}^{1} \log (z-x) d \mu_{k}(x), \quad z \in \mathbb{C} \backslash[0,1],
$$

where the branch of log is taken on the principal sheet, with a cut on $(-\infty, 0]$. Then

$$
\omega_{k}(z) \equiv g_{k}^{\prime}(z)=\int_{0}^{1} \frac{d \mu_{k}(x)}{z-x}, \quad z \in \mathbb{C} \backslash[0,1] .
$$

From equation (2.45) it follows that as $z \rightarrow \infty$,

$$
g_{k}(z)=\log z+O\left(z^{-1}\right)
$$

and from (2.46), that

$$
\omega_{k}(z)=\frac{1}{z}+O\left(z^{-2}\right)
$$

From equation (2.23) it follows that

$$
\omega_{k}(z)=\frac{V_{k}^{\prime}(z)}{2}-\sqrt{\frac{z-1}{z}} \frac{q_{k}(z)}{2},
$$

see, e.g., equations (3.27), (3.29) in [28], and

$$
l_{k}=2 g_{k}(1)-V_{k}(1) \text {. }
$$

Since

$$
\begin{aligned}
-g_{k}(1)=\lim _{u \rightarrow \infty}\left[g_{k}(u)-\log u-g_{k}(1)\right] & =\lim _{u \rightarrow \infty} \int_{1}^{u}\left[\omega_{k}(z)-\frac{1}{z}\right] d z \\
& =\int_{1}^{\infty}\left[\frac{V_{k}^{\prime}(z)}{2}-\sqrt{\frac{z-1}{z}} \frac{q_{k}(z)}{2}-\frac{1}{z}\right] d z,
\end{aligned}
$$

we obtain that

$$
g_{k}(1)=-\int_{1}^{\infty}\left[\frac{V_{k}^{\prime}(z)}{2}-\sqrt{\frac{z-1}{z}} \frac{q_{k}(z)}{2}-\frac{1}{z}\right] d z
$$

hence

$$
l_{k}=-\int_{1}^{\infty}\left[V_{k}^{\prime}(z)-\sqrt{\frac{z-1}{z}} q_{k}(z)-\frac{2}{z}\right] d z-V_{k}(1)
$$


We split the last integral as

$$
\begin{gathered}
\int_{1}^{\infty}\left[V_{k}^{\prime}(z)-\sqrt{\frac{z-1}{z}} q_{k}(z)-\frac{2}{z}\right] d z=\int_{1}^{\infty}\left[V_{k}^{\prime}(z)-4 b_{k}-\frac{1}{k z}\right] d z \\
-\int_{1}^{\infty}\left[\sqrt{\frac{z-1}{z}} q_{k}(z)+\frac{2}{z}-4 b_{k}-\frac{1}{k z}\right] d z=I_{1}-I_{2} .
\end{gathered}
$$

From (2.26) we have that

$$
I_{1}=\int_{1}^{\infty}\left[V_{k}^{\prime}(z)-4 b_{k}-\frac{1}{k z}\right] d z=-\int_{1}^{\infty} \frac{\gamma_{k} d z}{e^{\gamma_{k} k z}-1}=O\left(e^{-c_{0} k}\right), \quad c_{0}>0 .
$$

Let us evaluate $I_{2}$. By (2.44),

$$
I_{2}=\int_{1}^{\infty}\left[\sqrt{\frac{z-1}{z}} 4 b_{k}+\sqrt{\frac{z-1}{z}} \frac{a_{k}}{z}+\sqrt{\frac{z-1}{z}} r_{k}(z)+\frac{2}{z}-4 b_{k}-\frac{1}{k z}\right] d z .
$$

Since

$$
\int_{1}^{\infty}\left(\sqrt{\frac{z-1}{z}}-1+\frac{1}{2 z}\right) d z=\frac{1}{2}-\ln 2
$$

we obtain that

$$
I_{2}=b_{k}(2-4 \ln 2)+\int_{1}^{\infty}\left[\sqrt{\frac{z-1}{z}} \frac{a_{k}}{z}+\sqrt{\frac{z-1}{z}} r_{k}(z)-\frac{2 b_{k}-2}{z}-\frac{1}{k z}\right] d z .
$$

From estimate (2.40) we obtain that

$$
\int_{1}^{\infty} \sqrt{\frac{z-1}{z}} r_{k}(z) d z=O\left(k^{-5 / 2}\right)
$$

hence

$$
I_{2}=b_{k}(2-4 \ln 2)+\int_{1}^{\infty}\left[\sqrt{\frac{z-1}{z}} \frac{a_{k}}{z}-\frac{2 b_{k}-2+\frac{1}{k}}{z}\right] d z+O\left(k^{-5 / 2}\right) .
$$

From equation (2.49) we have that

$$
\omega_{k}(z)=\frac{V_{k}^{\prime}(z)}{2}-\frac{1}{2} \sqrt{\frac{z-1}{z}}\left[4 b_{k}+\frac{a_{k}}{z}+r_{k}(z)\right] .
$$

By equating terms of the order of $\frac{1}{z}$ on both sides, we obtain that

$$
1=\frac{1}{2 k}-\frac{a_{k}}{2}+b_{k}
$$

hence

$$
a_{k}=2 b_{k}-2+\frac{1}{k} .
$$

By substituting this expression into (2.60) we obtain that

$$
I_{2}=b_{k}(2-4 \ln 2)+\left(2 b_{k}-2+\frac{1}{k}\right) \int_{1}^{\infty}\left(\sqrt{\frac{z-1}{z}}-1\right) \frac{d z}{z}+O\left(k^{-5 / 2}\right) .
$$


Since

$$
\int_{1}^{\infty}\left(\sqrt{\frac{z-1}{z}}-1\right) \frac{d z}{z}=2 \ln 2-2
$$

we obtain that

$$
\begin{aligned}
I_{2} & =b_{k}(2-4 \ln 2)+\left(2 b_{k}-2+\frac{1}{k}\right)(2 \ln 2-2)+O\left(k^{-5 / 2}\right) \\
& =-2 b_{k}-4 \ln 2+4+\frac{2 \ln 2-2}{k}+O\left(k^{-5 / 2}\right) \\
& =2-4 \ln 2+\frac{2 \ln 2-1}{k}-\frac{\zeta\left(\frac{3}{2}\right)}{4 \sqrt{\pi(r-1)} k^{3 / 2}}+O\left(k^{-5 / 2}\right) .
\end{aligned}
$$

By (2.53), (2.54),

$$
l_{k}=I_{2}-I_{1}-V_{k}(1)
$$

and by (2.25) and (2.17),

$$
\begin{aligned}
V_{k}(1) & =4 b_{k}+\frac{\ln \left(4 b_{k} k\right)}{k}+O\left(k^{-5 / 2}\right) \\
& =4+\frac{\ln k}{k}+\frac{2 \ln 2-2}{k}+\frac{\zeta\left(\frac{3}{2}\right)}{2 \sqrt{\pi(r-1)} k^{3 / 2}}-\frac{1}{2 k^{2}}+O\left(k^{-5 / 2}\right)
\end{aligned}
$$

hence

$$
l_{k}=-2-4 \ln 2-\frac{\ln k}{k}+\frac{1}{k}-\frac{3 \zeta\left(\frac{3}{2}\right)}{4 \sqrt{\pi(r-1)} k^{3 / 2}}+\frac{1}{2 k^{2}}+O\left(k^{-5 / 2}\right)
$$

2.4. Evaluation of $h_{k, \alpha}$. According to Vanlessen's asymptotic formula, see [28],

$$
h_{k, \alpha}=\frac{\pi}{8} \beta_{k}^{2 k+2} e^{k l_{k}}\left[1+\left(\frac{3}{4 q_{k}(0)}+\frac{47}{12 q_{k}(1)}-\frac{q_{k}^{\prime}(1)}{4 q_{k}(1)^{2}}\right) \frac{1}{k}+O\left(k^{-2}\right)\right] .
$$

Observe that in [28] this formula is proved under the assumption that the weight for orthogonal polynomials has the form

$$
w(x)=x^{\gamma} e^{-Q(x)}, \quad \gamma>-1,
$$

where $Q(x)$ is a polynomial. In Appendix at the end of the paper we show what changes in the paper of Vanlessen [28] should be made to prove (2.70) for the weight $w_{\alpha}(x)$ given by formula (2.1). By (2.35),

$$
q_{k}(0)=4+O\left(k^{-1 / 2}\right), \quad q_{k}(1)=4+O\left(k^{-1 / 2}\right) .
$$

By (2.34),

$$
q_{k}^{\prime}(1)=-\frac{\gamma_{k}}{2 \pi i k^{1 / 2}} \oint_{k \Gamma} \sqrt{\frac{u}{(u / k)-1}} \frac{k d u}{\left(e^{\gamma_{k} u}-1\right)(u-k)^{2}},
$$

hence

$$
q_{k}^{\prime}(1)=O\left(k^{-1 / 2}\right)
$$


because by condition (2.31), the function $\frac{k}{u-k}$ is bounded by $1 / c$ for $u \in k \Gamma$. From (2.72) and (2.74) we obtain that

$$
1+\left(\frac{3}{4 q_{k}(0)}+\frac{47}{12 q_{k}(1)}-\frac{q_{k}^{\prime}(1)}{4 q_{k}(1)^{2}}\right) \frac{1}{k}=1+\frac{7}{6 k}+O\left(k^{-3 / 2}\right) .
$$

By substituting formulae (2.18), (2.17), (2.69), and (2.75) into (2.70) and by using the Stirling formula for $k$ !, we obtain that

$$
\ln \frac{h_{k, \alpha}}{(k !)^{2}}=-\frac{\zeta\left(\frac{3}{2}\right)}{2 \sqrt{\pi(r-1)} k^{1 / 2}}+\frac{1}{4 k}+O\left(k^{-3 / 2}\right)
$$

(we use MAPLE for this calculation). Theorem 1.1 is proved.

\section{Appendix A. Proof of Formula (2.70)}

We use the notations and results from the work [28] of Vanlessen. The essential difference with [28] is that we consider not a fixed but a shrinking neighborhood of the origin,

$$
\tilde{U}_{\delta, k}=\left\{z \in \mathbb{C}:|z| \leq \frac{\delta}{k}\right\},
$$

where $\delta>0$ is small enough so that the function $V_{k}(x)$ is analytic in $\tilde{U}_{\delta, k}$, see (2.19). As in [28], we consider a sequence of transformations of the Riemann-Hilbert problem for orthogonal polynomials, and in the end we arrive at the following Riemann-Hilbert problem on a $2 \times 2$ matrix-valued function $R(z)$ :

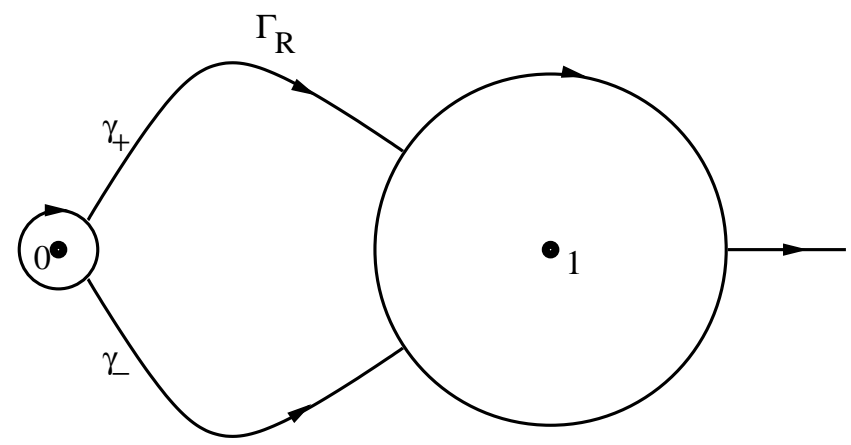

Figure 6. The contour $\Gamma_{R}$.

(1) $R(z)$ is analytic on $\mathbb{C} \backslash \Gamma_{R}$, where $\Gamma_{R}$ is the contour shown on Fig. 6, and it has limits, $R_{+}(z)$ and $R_{-}(z)$, on $\Gamma_{R}$, as $z$ approaches a point on $\Gamma_{R}$ from the left and from the right of the contour, with respect to the orientation indicated on Fig. 6.

(2) On $\Gamma_{R}, R(z)$ satisfies the jump condition, $R_{+}(z)=R_{-}(z) v_{R}(z)$, where $v_{R}(z)$ is an explicit matrix-valued function.

(3) $R(z) \simeq I+\frac{R_{1}}{z}+\ldots$ as $z \rightarrow \infty$, where $I$ is the identity matrix.

The contour $\Gamma_{R}$ consists of the circle $\partial \tilde{U}_{\delta, k}$, the circle $\partial U_{\delta}$, where

$$
U_{\delta}=\{z \in \mathbb{C}:|z-1| \leq \delta\},
$$


the boundaries of the lenses, $\gamma_{ \pm}$, and the semi-infinite interval $[1+\delta, \infty)$. The jump matrix $v_{R}$ on $\partial \tilde{U}_{\delta, k}$ has the following asymptotics as $k \rightarrow \infty$ :

$$
v_{R}(z) \simeq I+\sum_{n=1}^{\infty} \tilde{\Delta}_{n}(z) k^{-n}
$$

see formulae (3.105) and (3.98) in [28]. This asymptotic formula holds under the condition that $k^{2} z \rightarrow \infty$. Under this condition, for any $N \geq 1$ there exists a constant $C_{N}>0$ such that

$$
\left|v_{R}(z)-I-\sum_{n=1}^{N} \tilde{\Delta}_{n}(z) k^{-n}\right| \leq \frac{C_{N}}{\left(k^{2}|z|\right)^{\frac{N}{2}}|z|^{2}} .
$$

The condition $k^{2} z \rightarrow \infty$ is valid for $z \in \partial \tilde{U}_{\delta, k}$, and in this case the last estimate gives that

$$
\sup _{z \in \partial \tilde{U}_{\delta, k}}\left|v_{R}(z)-I-\sum_{n=1}^{N} \tilde{\Delta}_{n}(z) k^{-n}\right| \leq \frac{\tilde{C}_{N}}{k^{\frac{N}{2}-2}}, \quad \tilde{C}_{N}=\frac{C_{N}}{\delta^{\left[\frac{N}{2}\right]+2}} .
$$

The coefficients $\tilde{\Delta}_{n}(z)$ in $($ A.3 $)$ are given by the following formula:

$$
\tilde{\Delta}_{n}(z)=\frac{1}{\tilde{\phi}_{k}(z)^{n / 2}} P^{(\infty)}(z)(-z)^{\frac{1}{2} \sigma_{3}} A_{n}(-z)^{-\frac{1}{2} \sigma_{3}} P^{(\infty)}(z)^{-1}
$$

where

$$
\begin{aligned}
& P^{(\infty)}(z)=2^{-\sigma_{3}}\left(\begin{array}{ll}
\frac{a(z)+a(z)^{-1}}{2} & \frac{a(z)-a(z)^{-1}}{2 i} \\
\frac{a(z)-a(z)^{-1}}{-2 i} & \frac{a(z)+a(z)^{-1}}{2}
\end{array}\right)\left(\frac{2 z-1+2 \sqrt{z(z-1)}}{z}\right)^{\frac{1}{2} \sigma_{3}} \\
& a(z)=\left(\frac{z-1}{z}\right)^{1 / 4}, \quad \tilde{\phi}_{k}(z)=-\left[\frac{1}{4} \int_{0}^{z} \sqrt{\frac{1-s}{s}} q_{k}(s) d s\right]^{2}, \\
& A_{n}=\frac{\prod_{j=1}^{n}\left[4-(2 j-1)^{2}\right]}{16^{n} n !}\left(\begin{array}{cc}
\frac{(-1)^{n}}{4 n}(3+2 n) & \left(n-\frac{1}{2}\right) i \\
(-1)^{n+1}\left(n-\frac{1}{2}\right) i & \frac{1}{4 n}(3+2 n)
\end{array}\right),
\end{aligned}
$$

see equation (3.99) in [28]. The function $\tilde{\phi}_{k}(z)$ is analytic in $\tilde{U}_{\delta, k}$. From (2.29), (2.17), and (2.36) we obtain that as $k \rightarrow \infty$,

$$
\sup _{z \in \tilde{U}_{\delta, k}}\left|q_{k}(z)-4\right|=O\left(k^{-1 / 2}\right) .
$$

By (A.8) this implies that

$$
\sup _{z \in \tilde{U}_{\delta, k}}\left|\frac{\tilde{\phi}_{k}(z)}{\phi(z)}-1\right|=O\left(k^{-1 / 2}\right), \quad \phi(z)=-\left(\int_{0}^{z} \sqrt{\frac{1-s}{s}} d s\right)^{2}=-4 z+\frac{4 z^{2}}{3}+\ldots
$$

The function $\tilde{\Delta}_{n}(z)$ is meromorphic in $\tilde{U}_{\delta, k}$ with the only possible pole at the origin of the order at most $\left[\frac{n+1}{2}\right]$, see [28]. This result, combined with explicit formula (․․6), implies that there exists $c_{n}>0$ such that

$$
\sup _{z \in \partial \tilde{U}_{\delta, k}}\left|\tilde{\Delta}_{n}(z) k^{-n}\right| \leq c_{n} k^{-n+\left[\frac{n+1}{2}\right]} .
$$


This, in turn, allows us to improve estimate (A.5) as follows: for any $N \geq 1$ there exists $\tilde{c}_{N}>0$ such that

$$
\sup _{z \in \partial \tilde{U}_{\delta, k}}\left|v_{R}(z)-I-\sum_{n=1}^{N} \tilde{\Delta}_{n}(z) k^{-n}\right| \leq \tilde{c}_{N} k^{-N+\left[\frac{N}{2}\right]},
$$

so that the error term is estimated by a constant times the estimate of $\tilde{\Delta}_{N+1}(z) k^{-(N+1)}$. When $N=1$, this gives that

$$
\sup _{z \in \partial \tilde{U}_{\delta, k}}\left|v_{R}(z)-I-\frac{\tilde{\Delta}_{1}(z)}{k}\right|=O\left(k^{-1}\right)
$$

The function $\tilde{\Delta}_{1}(z)$ has a simple pole at 0 and its residue is equal to

$$
B_{k}=\frac{3}{16 q_{k}(0)} 2^{-\sigma_{3}}\left(\begin{array}{cc}
1 & i \\
i & -1
\end{array}\right) 2^{\sigma_{3}}
$$

see equation (4.11) in [28]. The function $\tilde{\Delta}_{1}(z)-\frac{B_{k}}{z}$ is regular at $z=0$ and from explicit formula (A.6) we obtain that as $k \rightarrow \infty$,

$$
\sup _{z \in \partial \tilde{U}_{\delta, k}}\left|\tilde{\Delta}_{1}(z)-\frac{B_{k}}{z}\right|=O(1)
$$

hence from (A.14) we obtain that

$$
\sup _{z \in \partial \tilde{U}_{\delta, k}}\left|v_{R}(z)-I-\frac{B_{k}}{k z}\right|=O\left(k^{-1}\right) .
$$

The problem here is that $v_{R}(z)$ is not close to $I$ on $\partial \tilde{U}_{\delta, k}$. We will overcome this obstacle by a transformation of the Riemann-Hilbert problem for $R(z)$.

Observe that

$$
\operatorname{Tr} B_{k}=0, \quad \operatorname{det} B_{k}=0,
$$

hence

$$
\operatorname{det}\left(I+\frac{B_{k}}{k z}\right)=1
$$

hence the matrix $I+\frac{B_{k}}{k z}$ is invertible for any $z \neq 0$. Let us make the substitution,

$$
R(z)=\left\{\begin{array}{l}
\tilde{R}(z), \quad z \in \tilde{U}_{\delta, k}, \\
\tilde{R}(z)\left(I+\frac{B_{k}}{k z}\right), \quad z \notin \tilde{U}_{\delta, k} .
\end{array}\right.
$$

Then $\tilde{R}(z)$ solves the Riemann-Hilbert problem similar to the one for $R(z)$, with the jump matrix $\tilde{v}_{R}(z)$ such that

$$
\tilde{v}_{R}(z)=v_{R}(z)\left(I+\frac{B_{k}}{k z}\right)^{-1}, \quad z \in \partial \tilde{U}_{\delta, k}
$$

and

$$
\tilde{v}_{R}(z)=\left(I+\frac{B_{k}}{k z}\right) v_{R}(z)\left(I+\frac{B_{k}}{k z}\right)^{-1}, \quad z \in \Gamma_{R} \backslash \partial \tilde{U}_{\delta, k}
$$


From (A.17) we obtain that

$$
\sup _{z \in \partial \tilde{U}_{\delta, k}}\left|\tilde{v}_{R}(z)-I\right|=O\left(k^{-1}\right)
$$

Also, since the equilibrium density function diverges as $z^{-1 / 2}$ at the origin, we obtain that $v_{R}(z)$ is sub-exponentially small on the boundary of lenses,

$$
\sup _{z \in \gamma_{+} \cup \gamma_{-}}\left|v_{R}(z)-I\right|=O\left(e^{-c \sqrt{k}}\right), \quad c>0 .
$$

This implies that $\tilde{v}_{R}(z)$ satisfies a similar estimate,

$$
\sup _{z \in \gamma_{+} \cup \gamma_{-}}\left|\tilde{v}_{R}(z)-I\right|=O\left(e^{-c \sqrt{k}}\right), \quad c>0 .
$$

In addition,

$$
\sup _{z \in \partial U_{\delta}}\left|\tilde{v}_{R}(z)-I\right|=O\left(k^{-1}\right)
$$

and

$$
\left|\tilde{v}_{R}(z)-I\right|=O\left(e^{-c k z}\right), \quad z \geq 1 ; \quad c>0 .
$$

These estimates of smallness of $\left(\tilde{v}_{R}(z)-I\right)$ on $\Gamma_{R}$ enable us to solve the Riemann-Hilbert problem for $\tilde{R}(z)$ by a series of perturbation theory. The fact that the radius of $\tilde{U}_{\delta, k}, r=\frac{\delta}{k}$, is tending to zero does not cause a problem, see appendix to the work [5] of Bleher and Kuijlaars.

The rest of the proof of formula (2.70) goes along the lines of [28]. Namely, by formula (4.17) in [28],

$$
h_{k, \alpha}=\frac{\pi}{8} \beta_{k}^{2 k+2} e^{k l_{k}}\left[1-16 i\left(R_{1}\right)_{12}+O\left(k^{-2}\right)\right] .
$$

By (A.20),

$$
\left(R_{1}\right)_{12}=\left(\tilde{R}_{1}\right)_{12}+\frac{\left(B_{k}\right)_{12}}{k}+O\left(k^{-2}\right)=\left(\tilde{R}_{1}\right)_{12}+\frac{3 i}{64 q_{k}(0) k}+O\left(k^{-2}\right)
$$

By applying formula (4.11) in [28] to $\tilde{R}_{1}$, we obtain that

$$
\left(\tilde{R}_{1}\right)_{12}=-\frac{q_{k}^{\prime}(1) i}{64 q_{k}(1)^{2} k}+\frac{47 i}{192 q_{k}(1) k}+O\left(k^{-2}\right) .
$$

Observe that the first term in formula (4.11) in [28 is missing in this case, because the function $\left[\tilde{\Delta}_{1}(z)-\frac{B_{k}}{z}\right]$ is regular at $z=0$. From the last two formulae we obtain that

$$
-16 i\left(R_{1}\right)_{12}=\left[\frac{3}{4 q_{k}(0)}-\frac{q_{k}^{\prime}(1)}{4 q_{k}(1)^{2}}+\frac{47}{12 q_{k}(1)}\right] \frac{1}{k}+O\left(k^{-2}\right) .
$$

By substituting this into (A.28) we obtain (2.70). 


\section{REFERENCES}

[1] D. Allison and N. Reshetikhin, Numerical study of the 6-vertex model with domain wall boundary conditions, Ann. Inst. Fourier (Grenoble) 55 (2005) 18471869.

[2] R. Baxter, Exactly solved models in statistical mechanics, Academic Press, San Diego, CA.

[3] M.T. Batchelor, R.J. Baxter, M.J. O'Rourke, and C.M. Yung, Exact solution and interfacial tension of the six-vertex model with anti-periodic boundary conditions. J. Phys. A 28 (1995) 2759-2770.

[4] P.M. Bleher and V.V. Fokin, Exact solution of the six-vertex model with domain wall boundary conditions. Disordered phase. Commun. Math. Phys. 268 (2006), 223-284.

[5] P.M. Bleher and A.B.J. Kuijlaars, Large n limit of Gaussian random matrices with external source, part III: Double scaling limit. Commun. Math. Phys. 270 (2007), 481-517.

[6] P.M. Bleher and K. Liechty, Exact solution of the six-vertex model with domain wall boundary conditions. Ferroelectric phase. arXiv:0712.4091 [math.ph].

[7] D.M. Bressoud, Proofs and Confirmations: the Story of the Alternating Sign Matrix Conjecture, published jointly by the Mathematical Association of America (Spectrum Series) and Cambridge University Press (or Cambridge University Press, NY) 1999.

[8] H. Cohn, N. Elkies and J. Propp, Local statistics for random domino tilings of the Aztec diamond, Duke Math. J. 85 (1996) 117166.

[9] F. Colomo and A.G. Pronko, Square ice, alternating sign matrices, and classical orthogonal polynomials, J. Stat. Mech. Theory Exp. 2005, no. 1, 005, 33 pp. (electronic).

[10] F. Colomo and A.G. Pronko, The arctic circle revisited. Preprint. arXiv:0704.0362,

[11] K. Eloranta, Diamond Ice, J. Statist. Phys. 96 (1999) 10911109.

[12] P.L. Ferrari and H. Spohn, Domino tilings and the six-vertex model at its free fermion point, J. Phys. A: Math. Gen. 39 (2006) 1029710306.

[13] A. G. Izergin, Partition function of the six-vertex model in a finite volume. (Russian) Dokl. Akad. Nauk SSSR 297 (1987), no. 2, 331-333; translation in Soviet Phys. Dokl. 32 (1987), 878-880.

[14] A. G. Izergin, D. A. Coker, and V. E. Korepin, Determinant formula for the six-vertex model. J. Phys. A, 25 (1992), 4315-4334.

[15] R. Kenyon and A. Okounkov, Limit shapes and the complex Burgers equation. Acta Math., 199 (2007), 263-302.

[16] V. E. Korepin, Calculation of norms of Bethe wave functions. Commun. Math. Phys. 86 (1982), 391-418.

[17] R. Kenyon, A. Okounkov, and S. Sheffield, Dimers and amoebae. Ann. of Math. 163 (2006), no. 3, $1019-1056$.

[18] V. Korepin and P. Zinn-Justin, Thermodynamic limit of the six-vertex model with domain wall boundary conditions, J. Phys. A 33 No. 40 (2000), 7053

[19] G. Kuperberg, Another proof of the alternating sign matrix conjecture. Int. Math. Res. Not. (1996), 139-150.

[20] E. H. Lieb, Exact solution of the problem of the entropy of two-dimensional ice. Phys. Rev. Lett. 18 (1967) 692.

[21] E. H. Lieb, Exact solution of the two-dimensional Slater KDP model of an antiferroelectric. Phys. Rev. Lett. 18 (1967) 1046-1048.

[22] E. H. Lieb, Exact solution of the two-dimensional Slater KDP model of a ferroelectric. Phys. Rev. Lett. 19 (1967) 108-110.

[23] E. H. Lieb, Residual entropy of square ice. Phys. Rev. 162 (1967) 162.

[24] E. H. Lieb and F. Y. Wu, Two dimensional ferroelectric models, in Phase Transitions and Critical Phenomena, C. Domb and M. Green eds., vol. 1, Academic Press (1972) 331-490.

[25] S. Sheffield, Random surfaces. Astérisque 304 (2005), vi+175 pp.

[26] O.F. Syljuasen and M.B. Zvonarev, Directed-loop Monte Carlo simulations of Vertex models, Phys. Rev. E70 (2004) 016118.

[27] B. Sutherland, Exact solution of a two-dimensional model for hydrogen-bonded crystals. Phys. Rev. Lett. 19 (1967), 103-104.

[28] M. Vanlessen, Strong asymptotics of Laguerre-type orthogonal polynomials and applications in random matrix theory, Construct. Approx. 25 (2007), 125-175. 
[29] F.Y. Wu and K.Y. Lin, Staggered ice-rule vertex model. The Pfaffian solution. Phys. Rev. B 12 (1975), 419-428.

[30] P. Zinn-Justin, Six-vertex model with domain wall boundary conditions and one-matrix model. Phys. Rev. E 62 (2000), 3411-3418.

[31] P. Zinn-Justin, The influence of boundary conditions in the six-vertex model. Preprint, arXiv:cond-mat/0205192.

Department of Mathematical Sciences, Indiana University-Purdue University IndianapoLis, 402 N. BlaCkFord St., Indianapolis, IN 46202, U.S.A.

E-mail address: bleher@math.iupui.edu

Department of Mathematical Sciences, Indiana University-Purdue University IndianapoLis, 402 N. Blackford St., Indianapolis, IN 46202, U.S.A.

E-mail address: kliechty@math.iupui.edu 